\title{
Perfil epidemiológico do suicídio no estado do Rio Grande do Sul, Brasil, de 2010 a 2016
}

\section{Epidemiological profile of suicide in Rio Grande do Sul/Brazil from 2010 to 2016}

\author{
Nathalia Fattah ${ }^{1,2,3}$ (D) Eduardo Viegas da Silva ${ }^{1}$ (D) Claudia Weyne Cruz ${ }^{4}$ (D), \\ Mayte Raya Amazarray ${ }^{3}$ (D) \\ 'Centro Estadual de Vigilância em Saúde, Secretaria de Estado da Saúde do Rio Grande do Sul - Porto Alegre (RS), Brasil. \\ ${ }^{2}$ Departamento de Ações em Saúde, Secretaria de Estado da Saúde do Rio Grande do Sul - Porto Alegre (RS), Brasil. \\ ${ }^{3}$ Programa de Pós-Graduação em Psicologia e Saúde, Universidade Federal de Ciências da Saúde de Porto Alegre - Porto Alegre \\ (RS), Brasil. \\ ${ }^{4}$ Escola de Saúde Pública, Secretaria de Estado da Saúde do Rio Grande do Sul - Porto Alegre (RS), Brasil.
}

Como citar: Fattah N, Silva EV, Cruz CW, Amazarray MR. Perfil epidemiológico do suicídio no estado do Rio Grande do Sul, Brasil, de 2010 a 2016. Cad Saúde Colet, 2021;29(4):561-574. https://doi.org/10.1590/1414-462X202129040017

\section{Resumo}

Introdução: A sistematização de informações sobre o fenômeno do suicídio é imprescindível para subsidiar o planejamento de ações e estratégias de intervenção. Objetivo: Descrever características epidemiológicas da mortalidade por suicídio no estado do Rio Grande do Sul de 2010 a 2016. Método: Os dados foram obtidos a partir do Sistema de Informação sobre Mortalidade e analisados segundo variáveis sociodemográficas. Foi utilizado o método de Prais-Winsten para estimar a variação média anual e indicar a tendência dos coeficientes. Resultados: Foram registrados 7.796 óbitos no período analisado, correspondendo a um coeficiente médio de 10,7 por 100 mil habitantes. Aproximadamente $80 \%$ dos suicídios foram de homens e $40 \%$ do total se concentrou entre 40 e 59 anos de idade. Os coeficientes mais altos foram encontrados entre pessoas idosas. Foi verificada tendência de mortalidade crescente para mulheres e decrescente para homens na faixa etária de 20 a 29 anos. Entre 10 e 14 anos de idade, foi identificada tendência crescente para o sexo feminino. Na média do período, os maiores coeficientes concentraram-se no centro e norte do estado. Conclusão: São sugeridos estudos aprofundados nas regiões e nos grupos prioritários apontados, a fim de subsidiar o aprimoramento de políticas públicas para a prevenção do suicídio.

Palavras-chave: suicídio; epidemiologia; prevenção; políticas públicas.

\begin{abstract}
Background: The systematization of information about the phenomenon of suicide is crucial to support action planning and intervention strategies. Objective: To describe the epidemiological characteristics of suicide mortality in the State of Rio Grande do Sul from 2010 to 2016. Method: The data were collected from the Mortality Information System and analyzed according to sociodemographic variables. The PraisWinsten method estimated the annual average variation and indicated the trend of mortality coefficients. Results: We found 7,796 deaths recorded in the period, corresponding to an average coefficient of 10.7 per 100 thousand inhabitants. Approximately $80 \%$ of the suicides were of men, and $40 \%$ of the total were concentrated between the ages of 40 and 59. The highest coefficients were found among the elderly population. There was a trend of increasing mortality for women and decreasing for men in the age group of 20 to 29. An increasing trend was identified for females between the ages of 10 and 14. On average, the highest coefficients were concentrated in the center and north of the state. Conclusion:We suggest in-depth studies to be carried out in the regions and priority groups mentioned to help improve public policies for suicide prevention.
\end{abstract}

Keywords: suicide; epidemiology; prevention; public policies.

Este é um artigo publicado em acesso aberto (Open Access) sob a licença Creative Commons Attribution, que permite uso, distribuição e reprodução em qualquer meio, sem restrições desde que o trabalho original seja corretamente citado.
Trabalho realizado na Universidade Federal de Ciências da Saúde de Porto Alegre (UFCSPA) - Porto Alegre (RS), Brasil. Correspondência: Nathalia Fattah. E-mail: nathalia-fattah@saude.rs.gov.br

Fonte de financiamento: nenhuma.

Conflito de Interesses: nada a declarar.

Recebido em: Jan. 17, 2019. Aprovado em: Ago. 10, 2020 


\section{INTRODUÇÃO}

Suicídio é o ato humano de infligir a si próprio o fim da vida e, por existir em todas as sociedades já conhecidas e estudadas, é considerado um fenômeno universal' ${ }^{1}$. Na variedade de conceitos existentes, a intenção de morrer é o elemento-chave². Definido pela Organização Mundial da Saúde (OMS) como o ato deliberado de tirar a própria vida, é resultante da interação de fatores pessoais, psicológicos, biológicos, sociais, culturais e ambientais ${ }^{3}$. Esse fenômeno tão complexo compreende um amplo espectro de manifestações, que vão desde pensamentos autodestrutivos, ideação, plano, tentativa até o suicídio consumado ${ }^{1,3}$, que pode ser visto como a única maneira de cessar uma dor psíquica intolerável para pessoas que se encontram em situações-limite ${ }^{4}$.

A partir da década de 1990, o suicídio passou a ser considerado um problema de saúde pública ${ }^{4}$. Segundo dados da OMS de 2014³ ${ }^{3}$ uma pessoa tira a própria vida a cada 40 segundos. O coeficiente mundial no ano de 2012 foi estimado em 11,4 por 100 mil habitantes (15 para homens e 8 para mulheres), o que equivale a mais de 800 mil mortes ${ }^{3}$. Em geral, as taxas são maiores na faixa etária acima dos 70 anos. Entre os jovens de 15 a 29 anos de idade, o suicídio é a segunda causa de morte 3 .

Embora os coeficientes ajustados por idade sejam levemente maiores em nações desenvolvidas (12,7 versus 11,2 por 100 mil habitantes), mais de $75 \%$ dos suicídios acontecem em países subdesenvolvidos ou em desenvolvimento ${ }^{3}$. No Brasil, que ocupa a oitava posição em números absolutos ${ }^{3}$, ocorrem cerca de 11 mil mortes a cada ano ${ }^{5}$. Conforme dados do Ministério da Saúde (MS) ${ }^{6}$, o coeficiente de óbitos por essa causa foi estimado em 5,7 por 100 mil habitantes em 2015, sendo aproximadamente quatro vezes maior entre os homens em relação às mulheres. O estado do Rio Grande do Sul (RS) tem, historicamente, taxas quase duas vezes maiores que a brasileira. Entre 2011 e 2015, apresentou o coeficiente mais alto (10,3 por 100 mil habitantes), seguido por Santa Catarina e Mato Grosso do Sul 6 .

Os dados sobre mortalidade do país estão disponíveis por meio do Sistema de Informação sobre Mortalidade (SIM) do Departamento de Informática do Sistema Único de Saúde (DATASUS). Esse sistema é alimentado pelas secretarias municipais de saúde, a partir de informações que constam nas declarações de óbito, e transmitido às esferas estadual e federal, que consolidam e disponibilizam os dados ${ }^{7}$. No entanto, é consenso que haja sub-registro dos óbitos por suicídio no Brasil e no mundo 2,3,4. Muitos deles são categorizados como acidentes (de trânsito, por exemplo) e eventos de intenção não determinada (como quedas e afogamentos), além das causas indefinidas. Minayo ${ }^{1}$ também destaca as interferências de ordem cultural, religiosa e moral no registro dos casos de morte autoinfligida. Essa conjuntura impede que se tenha uma real dimensão do problema que, seguramente, atinge patamares mais elevados do que revelam as informações oficiais.

Para cada suicídio, estima-se que entre 5 e 10 pessoas sejam gravemente afetadas emocional, social e economicamente pela perda: são os chamados sobreviventes. Em geral, trata-se de familiares, amigos e colegas próximos que relatam sentimentos mais frequentes de responsabilidade pela morte, rejeição, abandono, estigmatização, vergonha e constrangimento do que aqueles que perdem alguém por causas distintas ${ }^{8}$. Também são impactadas pessoas que presenciaram a situação de morte ou que atenderam à ocorrência, como policiais e profissionais de saúde. Os efeitos de um suicídio podem ainda reverberar durante gerações por meio da história familiar e do legado cultural de uma comunidade9.

Diante da magnitude do fenômeno, uma das metas do Plano de Ação em Saúde Mental 2013-2020 da OMS ${ }^{10}$, do qual o Brasil é signatário, é a redução em 10\% das taxas de suicídio até 2020. Na América Latina, o país foi o primeiro a apresentar Diretrizes Nacionais de Prevenção do Suicídio, em 2006 ${ }^{11}$, por meio da Portaria no 1.876 do MS ${ }^{12}$. Mais de 10 anos depois, o MS lançou uma Agenda de Ações Estratégicas para Vigilância e Prevenção do Suicídio, que considera os determinantes sociais da saúde e as especificidades de populações e grupos sociais em situação de maior vulnerabilidade a esse fenômeno ${ }^{13}$.

No RS, em dezembro de 2016, foi instituído o Comitê Estadual de Promoção da Vida e Prevenção do Suicídio por meio de decreto do governado ${ }^{14}$, sendo coordenado pela Secretaria de Estado da Saúde e composto por representantes de outros setores, incluindo a sociedade 
civil. O Comitê tem como uma de suas competências a elaboração de um Plano Estadual de Promoção da Vida e Prevenção do Suicídio.

A sistematização de informações sobre o fenômeno do suicídio é imprescindível para subsidiar o planejamento de ações e estratégias de intervenção. Por reconhecer essa necessidade, o presente estudo teve como objetivo descrever as características epidemiológicas da mortalidade por suicídio no RS em uma série histórica de sete anos.

\section{MÉTODO}

Trata-se de estudo epidemiológico, observacional, retrospectivo e descritivo a partir de dados secundários do SIM. Foram incluídos todos os óbitos de residentes do estado do RS codificados como lesão autoprovocada intencionalmente (categorias de X60 a X84) de acordo com a 10 Revisão da Classificação Internacional de Doenças (CID-10) ${ }^{15}$ no período de 2010 a 2016 (último ano com base de dados consolidada disponível).

Os dados do SIM foram coletados por meio da ferramenta Tabnet no site da Secretaria de Estado da Saúde do $\mathrm{RS}^{16}$. Como base para o cálculo dos coeficientes, foi utilizada a população residente acima de 5 anos de idade estimada pela Rede Interagencial de Informações para a Saúde (RIPSA $)^{17}$ para cada ano da série histórica. A população referente ao ano de 2015, última disponível, foi adotada como denominador também para o ano de 2016. Especificamente para a variável raça/cor, foi utilizada a população informada pelo Instituto Brasileiro de Geografia e Estatística (IBGE) ${ }^{18}$ referente ao ano de 2010, por ser a única disponibilizada para essa variável.

\section{Análise dos dados}

Os dados gerados foram exportados para os softwares Microsoft Excel e Statistical Package for the Social Sciences (SPSS), versão 25. Foram realizadas análises estatísticas descritivas (frequências e proporções) para estudar as variáveis sexo, faixa etária, raça/cor, escolaridade, situação conjugal, mês do óbito, método de perpetração e local de ocorrência da morte. É possível notar que não há informações sobre gênero nas declarações de óbito. Nos resultados e na discussão, foram utilizados os termos "branca", "preta", "indígena", "parda" e "amarela" conforme a classificação de raça/cor adotada pelos sistemas de informação consultados (SIM e IBGE).

Os diferentes métodos de perpetração do suicídio foram agrupados nas categorias: autointoxicação por drogas e medicamentos (de X60 a X65), autointoxicação por pesticidas, solventes, gases e vapores, outros produtos e substâncias não especificados (de X66 a X69), enforcamento (X70), arma de fogo (de X72 a X74), precipitação de local elevado (X80) e meios não especificados (X84). Foi feita a opção por agrupar na categoria "Outros" os métodos de perpetração que, somados, representaram apenas 4,2\% do total (X71, de X75 a X79 e de X81 a X83).

Para a estimativa dos coeficientes, o número absoluto de óbitos foi dividido pela população de interesse do ano correspondente, multiplicando-se o resultado por 100.000. Foram calculados coeficientes para as variáveis sexo, faixa etária, raça/cor e região de saúde.

Região de saúde é definida como

um espaço geográfico contínuo constituído por agrupamento de municípios limítrofes, delimitado a partir de identidades culturais, econômicas e sociais e de redes de comunicação e infraestrutura de transportes compartilhados com a finalidade de integrar a organização, o planejamento e a execução de ações e serviços de saúde. (Art.2 ${ }^{\circ}$ inciso I) ${ }^{19}$.

O RS, estado com população estimada em mais de 11 milhões de habitantes ${ }^{20}$, possui 497 municípios agrupados em 30 regiões de saúde ${ }^{21}$. Para calcular e comparar os coeficientes dos 30 territórios, os dados foram padronizados por idade e sexo por meio do método direto ${ }^{22}$, utilizando a população do estado, para cada ano analisado, como referência. Destaca-se que todas as regiões de saúde do RS têm população acima de 100 mil habitantes. 
O método de Prais-Winsten, modelo de regressão linear generalizada indicado para a análise de séries temporais de dados populacionais ${ }^{23}$, foi adotado para estimar a variação média anual (com intervalo de confiança de 95\%) e indicar a tendência dos coeficientes de mortalidade (crescente, decrescente ou estacionária) em relação às variáveis população total, sexo, faixa etária e região de saúde. Foi realizada a combinação das variáveis população e número de óbitos, resultando na variável derivada "coeficiente anual de mortalidade por suicídio por 100 mil habitantes". Esse coeficiente entrou como variável explanatória no modelo.

\section{RESULTADOS}

Foram registrados 7.796 óbitos por suicídio no estado do RS no período analisado, variando de 1.035, no ano de 2010, a 1.170, em 2016. Na Tabela 1, são apresentadas as frequências e proporções dos óbitos por sexo, de acordo com as seguintes variáveis: faixa etária, raça/cor, escolaridade, situação conjugal, método de perpetração e local de ocorrência da morte.

É possível destacar que $79,4 \%$ dos suicídios foram de homens. Em relação à faixa etária, a maior concentração de casos foi entre 40 e 59 anos de idade, em ambos os sexos, correspondendo a quase $40 \%$ de todas as mortes.

A população branca acumulou $90,5 \%$ dos óbitos e teve o maior coeficiente, com 11,33 por 100 mil habitantes. A população preta correspondeu a 3,9\% dos suicídios e teve um coeficiente de 7,46 por 100 mil habitantes. Já os indígenas representaram apenas $0,2 \%$ das mortes do período, com um coeficiente de 5,6 por 100 mil habitantes.

Foi observado que as mortes predominaram entre pessoas solteiras, viúvas ou separadas (56\%). No que se refere à escolaridade, é preciso ressaltar o alto índice de "Não informada" $(8,1 \%)$ e"Ignorada" (34,3\%). Entre os casos informados, quase 38\% tinham até sete anos de estudo.

Quanto ao método de perpetração, o enforcamento foi o mais frequente para ambos os sexos (73,4\% dos homens e $61,9 \%$ das mulheres), seguido de arma de fogo para os homens $(16,9 \%)$ e de drogas e medicamentos para as mulheres (10,5\%). A maior parte dos óbitos ocorreu no domicílio, para ambos os sexos (68,3\%). Mais de $19 \%$ das mulheres faleceram no hospital, sendo este o local de ocorrência para apenas 9,9\% dos homens.

Também foram analisados os dados quanto ao mês de ocorrência do óbito. Os meses de outubro a março tiveram frequências de suicídio acima da média do período estudado $(649,66 \pm 41,98)$, com destaque para dezembro e janeiro, que registraram 708 e 730 casos, respectivamente.

Na Figura 1, são apresentados os coeficientes totais de mortalidade por suicídio, que variaram de 10,1 (16,5 para homens e 3,9 para mulheres), em 2010, a 11,1 (17,9 para homens

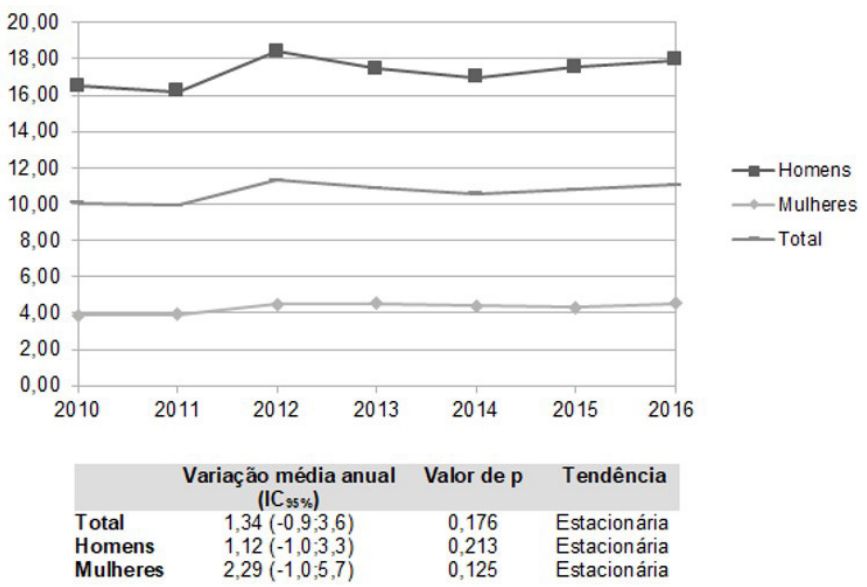

Figura 1. Coeficientes anuais de mortalidade por suicídio (por 100 mil habitantes) e percentual de variação média anual (intervalo de confiança de 95\%) segundo o modelo de regressão de Prais-Winsten, total e por sexo, Rio Grande do Sul, 2010-2016. Modelo de regressão de Prais-Winsten. Variável resposta: coeficiente anual de mortalidade por suicídio (por 100 mil habitantes). Variável explicativa: série temporal em anos, 2010-2016 
Tabela 1. Distribuição de óbitos por suicídio segundo o sexo, de acordo com a faixa etária, a raça/cor, a escolaridade, a situação conjugal, o método de perpetração e o local de ocorrência do óbito, Rio Grande do Sul, 2010-2016

\begin{tabular}{|c|c|c|c|c|c|c|}
\hline & \multicolumn{2}{|c|}{ Homens } & \multicolumn{2}{|c|}{ Mulheres } & \multicolumn{2}{|c|}{ Total } \\
\hline & $\mathbf{n}$ & $\%$ & $\mathbf{n}$ & $\%$ & $\mathbf{n}$ & $\%$ \\
\hline Total & 6.191 & 79,4 & 1.605 & 20,6 & 7.796 & 100 \\
\hline \multicolumn{7}{|l|}{ Faixa etária (anos) } \\
\hline 5 a 9 & 0 & 0 & 0 & 0 & 0 & 0 \\
\hline 10 a 14 & 27 & 0,4 & 27 & 1,7 & 54 & 0,67 \\
\hline 15 a 19 & 259 & 4,2 & 73 & 4,5 & 332 & 4,3 \\
\hline 20 a 29 & 912 & 14,7 & 207 & 12,9 & 1.119 & 14,4 \\
\hline 30 a 39 & 990 & 16,0 & 255 & 15,9 & 1.245 & 16,0 \\
\hline 40 a 49 & 1.167 & 18,8 & 333 & 20,7 & 1.500 & 19,2 \\
\hline 50 a 59 & 1.167 & 18,8 & 321 & 20,0 & 1.488 & 19,1 \\
\hline 60 a 69 & 837 & 13,5 & 210 & 13,1 & 1.047 & 13,4 \\
\hline 70 a 79 & 576 & 9,3 & 123 & 7,7 & 699 & 9,0 \\
\hline 80 e mais & 253 & 4,1 & 55 & 3,4 & 308 & 4,0 \\
\hline Ignorada & 3 & 0,0 & 1 & 0,1 & 4 & 0,1 \\
\hline \multicolumn{7}{|l|}{ Raça/cor } \\
\hline Branca & 5.579 & 90,1 & 1.477 & 92,0 & 7.056 & 90,5 \\
\hline Preta & 257 & 4,2 & 50 & 3,1 & 307 & 3,9 \\
\hline Indígena & 11 & 0,2 & 2 & 0,1 & 13 & 0,2 \\
\hline Parda & 294 & 4,7 & 56 & 3,5 & 350 & 4,5 \\
\hline Amarela & 3 & 0,0 & 2 & 0,1 & 5 & 0,1 \\
\hline Não informado & 47 & 0,8 & 18 & 1,1 & 65 & 0,8 \\
\hline \multicolumn{7}{|l|}{ Escolaridade (anos) } \\
\hline Nenhuma & 159 & 2,6 & 40 & 2,5 & 199 & 2,6 \\
\hline 1 a 3 & 874 & 14,1 & 218 & 13,6 & 1.092 & 14,0 \\
\hline 4 a 7 & 1.354 & 21,9 & 310 & 19,3 & 1.664 & 21,3 \\
\hline 8 a 11 & 881 & 14,2 & 269 & 16,8 & 1.150 & 14,8 \\
\hline 12 e mais & 255 & 4,1 & 131 & 8,2 & 386 & 5,0 \\
\hline Não informada & 495 & 8,0 & 139 & 8,7 & 634 & 8,1 \\
\hline Ignorada & 2.173 & 35,1 & 498 & 31,0 & 2.671 & 34,3 \\
\hline \multicolumn{7}{|l|}{ Situação conjugal } \\
\hline Solteiros, viúvos, separados & 3.463 & 55,9 & 905 & 56,4 & 4.368 & 56,0 \\
\hline Casados/união estável & 2.075 & 33,5 & 536 & 33,4 & 2.611 & 33,5 \\
\hline Em branco & 360 & 5,8 & 90 & 5,6 & 450 & 5,8 \\
\hline Ignorada & 293 & 4,7 & 74 & 4,6 & 367 & 4,7 \\
\hline \multicolumn{7}{|l|}{ Método de perpetração } \\
\hline Enforcamento & 4.543 & 73,4 & 993 & 61,9 & 5.536 & 71,0 \\
\hline Arma de fogo & 1.046 & 16,9 & 149 & 9,3 & 1.195 & 15,3 \\
\hline Autointoxicação por drogas e medicamentos & 134 & 2,2 & 168 & 10,5 & 302 & 3,9 \\
\hline $\begin{array}{l}\text { Autointoxicação por pesticidas, solventes, gases } \\
\text { e vapores, outros produtos e substâncias não } \\
\text { especificados }\end{array}$ & 123 & 2,0 & 94 & 5,9 & 217 & 2,8 \\
\hline Precipitação de local elevado & 106 & 1,7 & 73 & 4,6 & 179 & 2,3 \\
\hline Outros & 211 & 3,4 & 115 & 7,2 & 326 & 4,2 \\
\hline Meios não especificados & 27 & 0,4 & 13 & 0,8 & 40 & 0,5 \\
\hline \multicolumn{7}{|l|}{ Local ocorrência } \\
\hline Domicílio & 4.213 & 68,1 & 1.111 & 69,2 & 5.324 & 68,3 \\
\hline Hospital & 613 & 9,9 & 307 & 19,1 & 920 & 11,8 \\
\hline Via pública & 410 & 6,6 & 53 & 3,3 & 436 & 5,9 \\
\hline Outros estabelecimentos de saúde & 23 & 0,4 & 4 & 0,2 & 27 & 0,3 \\
\hline Outros & 907 & 14,7 & 126 & 7,9 & 1.033 & 13,3 \\
\hline Ignorado & 25 & 0,4 & 4 & 0,2 & 29 & 0,4 \\
\hline
\end{tabular}

Fonte: Sistema de Informação de Mortalidade (2018) 
e 4,5 para mulheres), em 2016, correspondendo a uma média de 10,7 no período. Os maiores valores foram encontrados no ano de 2012, chegando a 11,3 (18,4 para homens e 4,5 para mulheres). Os coeficientes do sexo masculino foram, em média, quatro vezes maiores do que os do sexo feminino, elevando a magnitude na população total. O modelo de regressão de Prais-Winsten mostrou tendência de mortalidade estacionária para ambos os sexos.

No que diz respeito à faixa etária, foi verificada elevação dos coeficientes de suicídio conforme o aumento da idade (Figura 2). Especialmente entre os homens, os coeficientes mais altos foram encontrados acima dos 70 anos de idade, com aumento expressivo na faixa etária de 70 a 79 anos em relação à anterior. Esse comportamento não se repetiu para mulheres, cuja mortalidade deixou de seguir um padrão de crescimento associado à faixa etária a partir dos 30 anos de idade.

Foi constatada tendência de aumento da mortalidade entre as mulheres e diminuição entre os homens na faixa etária de 20 a 29 anos (Tabela 2). Na faixa etária de 10 a 14 anos, foi identificada tendência de aumento para o sexo feminino.

Quanto à distribuição dos coeficientes de mortalidade entre as 30 regiões de saúde, foi verificado que, na média do período analisado, os maiores valores se concentraram no centro e norte do estado (Figura 3).

Conforme apresentado na Tabela 3, as regiões 7 (Vale dos Sinos) e 22 (Pampa) revelaram tendência de aumento nos coeficientes de mortalidade.

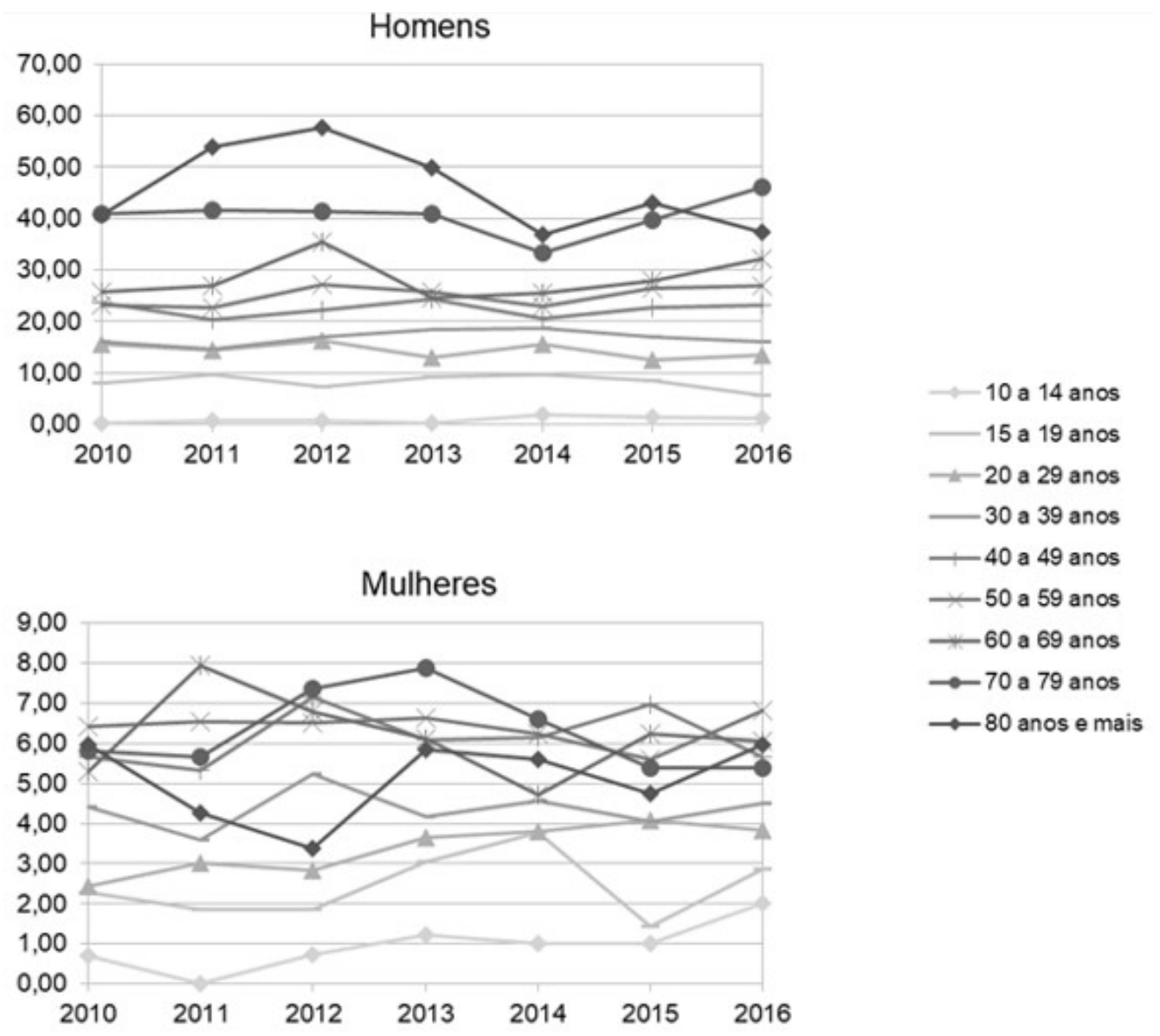

Figura 2. Coeficientes anuais de mortalidade por suicídio, segundo o sexo e a faixa etária, Rio Grande do Sul, 2010-2016 (coeficientes por 100 mil habitantes). Nota: uma vez que os coeficientes apresentaram grande variação entre homens e mulheres, foi necessário ajustar as escalas do eixo vertical dos gráficos para melhor expressão dos resultados. As linhas referentes à faixa etária de 5 a 9 anos foram suprimidas, por apresentarem taxas zeradas em todos os anos do período 
Tabela 2. Porcentagem de variação média anual (intervalo de confiança de $95 \%$ ) e tendências dos coeficientes de mortalidade por suicídio, segundo o modelo de regressão de Prais-Winsten, por sexo e faixa etária, Rio Grande do Sul, 2010-2016

\begin{tabular}{|c|c|c|c|c|c|c|}
\hline \multirow[b]{2}{*}{$\begin{array}{l}\text { Faixa } \\
\text { etária } \\
\text { (anos) }\end{array}$} & \multicolumn{3}{|c|}{ Homens } & \multicolumn{3}{|c|}{ Mulheres } \\
\hline & $\begin{array}{c}\text { Variação } \\
\text { média } \\
\text { anual } \\
\left(\text { IC }_{95 \%}\right)\end{array}$ & Valor de $p$ & Tendência & $\begin{array}{c}\text { Variação } \\
\text { média } \\
\text { anual } \\
\left(\text { IC }_{95 \%}\right)\end{array}$ & Valor de $p$ & Tendência \\
\hline 10 a 14 & $\begin{array}{c}30,28 \\
(-1,1 ; 71,6)\end{array}$ & 0,056 & Estacionária & $\begin{array}{c}15,02 \\
(4,8 ; 26,2)\end{array}$ & $0,014^{*}$ & Crescente $^{* *}$ \\
\hline 15 a 19 & $\begin{array}{c}-3,18 \\
(-12,4 ; 7,0)\end{array}$ & 0,420 & Estacionária & $\begin{array}{c}3,06 \\
(-12,8 ; 21,9)\end{array}$ & 0,644 & Estacionária \\
\hline 20 a 29 & $\begin{array}{c}-2,62(-4,2 ;- \\
1,0)\end{array}$ & $0,011^{*}$ & Decrescente & $\begin{array}{c}8,56 \\
(4,6 ; 12,7)\end{array}$ & $0,004^{*}$ & Crescente \\
\hline 30 a 39 & $\begin{array}{c}1,02 \\
(-4,7 ; 7,1)\end{array}$ & 0,652 & Estacionária & $\begin{array}{c}0,73 \\
(-3,1 ; 4,7)\end{array}$ & 0,630 & Estacionária \\
\hline 40 a 49 & $\begin{array}{c}0,54 \\
(-2,3 ; 3,4)\end{array}$ & 0,627 & Estacionária & $\begin{array}{c}1,86 \\
(-2,8 ; 6,8)\end{array}$ & 0,339 & Estacionária \\
\hline 50 a 59 & $\begin{array}{c}2,01 \\
(-1,6 ; 5,8)\end{array}$ & 0,200 & Estacionária & $\begin{array}{c}-1,07 \\
(-3,9 ; 1,8)\end{array}$ & 0,358 & Estacionária \\
\hline 60 a 69 & $\begin{array}{c}1,02 \\
(-6,0 ; 8,6)\end{array}$ & 0,716 & Estacionária & $\begin{array}{c}-2,33 \\
(-10,7 ; 6,8)\end{array}$ & 0,504 & Estacionária \\
\hline 70 a 79 & $\begin{array}{c}0,18 \\
(-5,9 ; 6,7) \\
\end{array}$ & 0,941 & Estacionária & $\begin{array}{c}-1,48 \\
(-12,2 ; 10,5) \\
\end{array}$ & 0,736 & Estacionária \\
\hline 80 e mais & $\begin{array}{c}-4,13 \\
(-13,0 ; 5,6)\end{array}$ & 0,293 & Estacionária & $\begin{array}{c}3,05 \\
(-8,2 ; 15,7)\end{array}$ & 0,512 & Estacionária \\
\hline
\end{tabular}

A faixa etária de 5 a 9 anos foi suprimida da análise por não registrar nenhuma morte no período analisado. Modelo de regressão de Prais-Winsten. Variável resposta: coeficiente anual de mortalidade por suicídio (por 100 mil habitantes). Variável explicativa: série temporal em anos, 2010-2016; * $p<0,05$; **a taxa de 2011 foi estimada a partir da média das taxas de 2010 e 2012, uma vez que foi zerada naquele ano, o que impossibilitaria a aplicação da função logarítmica e análise de regressão

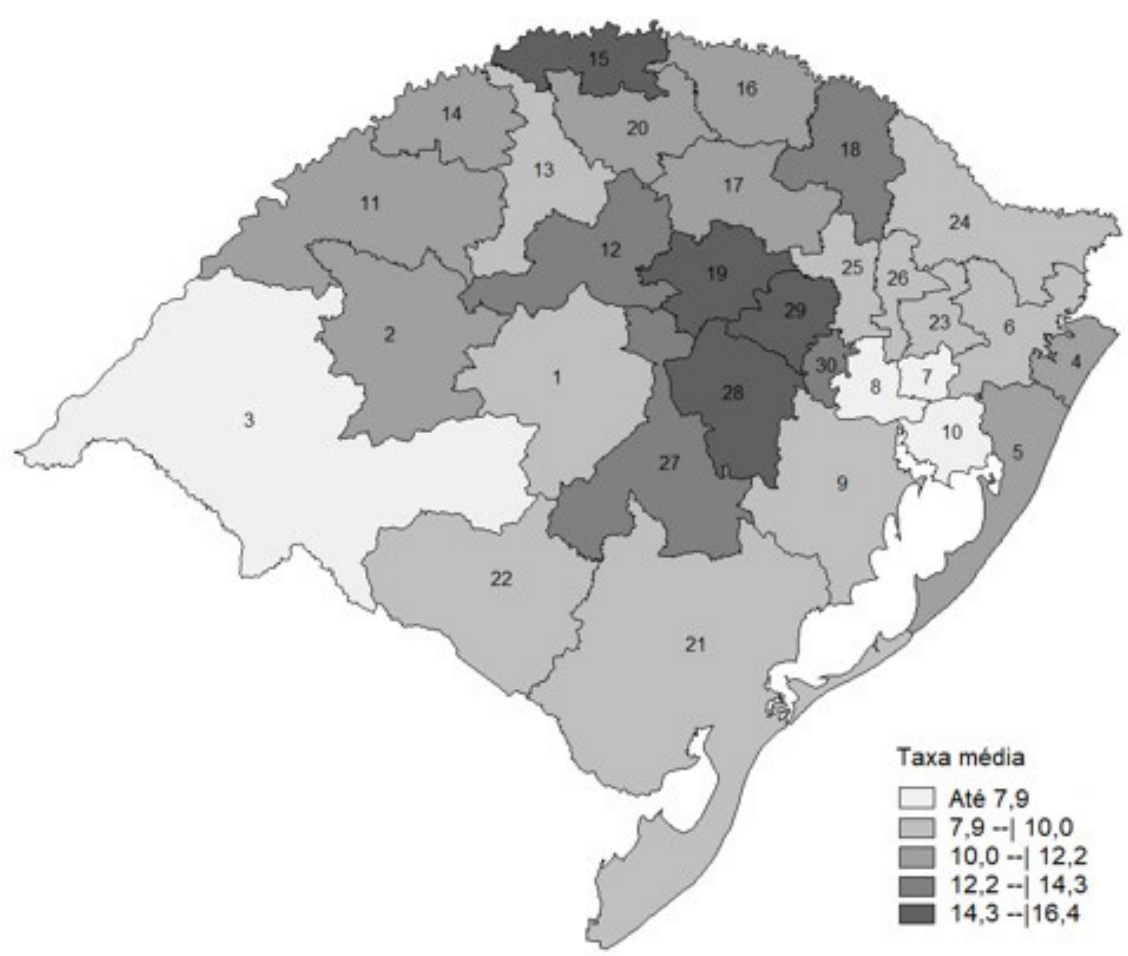

Figura 3. Média dos coeficientes de óbito por suicídio por região de saúde do Rio Grande do Sul, 2010-2016 (coeficientes por 100 mil habitantes, padronizados por faixa etária e sexo) 
Tabela 3. Percentual de variação média anual (intervalo de confiança de 95\%) e tendências dos coeficientes de mortalidade por suicídio, segundo o modelo de regressão de Prais-Winsten, por região de saúde, Rio Grande do Sul, 2010-2016

\begin{tabular}{|c|c|c|c|}
\hline Região de saúde & $\begin{array}{c}\text { Variação média } \\
\text { anual (IC } \\
95 \%\end{array}$ & Valor de $p$ & Tendência \\
\hline 1 Verdes Campos & $3,95(-2,0 ; 10,2)$ & 0,141 & Estacionária \\
\hline 2 Entre-Rios & $-1,79(-13,6 ; 11,7)$ & 0,716 & Estacionária \\
\hline 3 Fronteira Oeste & $1,89(-2,4 ; 6,4)$ & 0,294 & Estacionária \\
\hline 4 Belas Praias & $-2,06(-9,4 ; 5,9)$ & 0,502 & Estacionária \\
\hline 5 Bons Ventos & $8,28(-7,4 ; 26,7)$ & 0,232 & Estacionária \\
\hline $\begin{array}{l}6 \text { Vale do Paranhana e Costa } \\
\text { Serra }\end{array}$ & $-3,15(-12,0 ; 6,6)$ & 0,408 & Estacionária \\
\hline 7 Vale dos Sinos & $6,05(2,3 ; 10,0)$ & $0,011^{*}$ & Crescente \\
\hline 8 Vale do Caí e Metropolitana & $3,70(-0,6 ; 8,2)$ & 0,074 & Estacionária \\
\hline 9 Carbonífera/Costa Doce & $-0,81(-8,1 ; 7,1)$ & 0,783 & Estacionária \\
\hline 10 Capital e Vale do Gravataí & $3,55(-1,8 ; 9,2)$ & 0,145 & Estacionária \\
\hline 11 Sete Povos das Missões & $2,04(-6,8 ; 11,8)$ & 0,572 & Estacionária \\
\hline 12 Portal das Missões & $-3,61(-16,8 ; 11,7)$ & 0,526 & Estacionária \\
\hline 13 Região da Diversidade & $-1,83(-14,1 ; 12,2)$ & 0,721 & Estacionária \\
\hline 14 Fronteira Noroeste & $3,76(-2,5 ; 10,4)$ & 0,173 & Estacionária \\
\hline 15 Caminho das Águas & $-4,11(-9,2 ; 1,3)$ & 0,100 & Estacionária \\
\hline 16 Alto Uruguai Gaúcho & $-3,30(-14,6 ; 9,5)$ & 0,494 & Estacionária \\
\hline 17 Região do Planalto & $5,98(-0,8 ; 13,3)$ & 0,072 & Estacionária \\
\hline 18 Região das Araucárias & $-4,60(-15,6 ; 7,9)$ & 0,347 & Estacionária \\
\hline 19 Região do Botucaraí & $4,59(-8,2 ; 19,2)$ & 0,395 & Estacionária \\
\hline 20 Rota da Produção & $2,35(-2,3 ; 7,2)$ & 0,234 & Estacionária \\
\hline 21 Região Sul & $4,08(-3,3 ; 12,0)$ & 0,206 & Estacionária \\
\hline 22 Pampa & $9,92(5,3 ; 14,7)$ & $0,004^{*}$ & Crescente \\
\hline 23 Caxias e Hortências & $2,00(-12,1 ; 18,3)$ & 0,730 & Estacionária \\
\hline 24 Campos de Cima da Serra & $-6,36(-21,8 ; 12,2)$ & 0,370 & Estacionária \\
\hline 25 Vinhedos e Basalto & $-3,76(-10,5 ; 3,4)$ & 0,214 & Estacionária \\
\hline 26 Uva e Vale & $-3,25(-11,8 ; 6,1)$ & 0,377 & Estacionária \\
\hline 27 Jacuí Centro & $1,41(-7,7 ; 11,4)$ & 0,701 & Estacionária \\
\hline 28 Vale do Rio Pardo & $-4,02(-17,3 ; 11,4)$ & 0,487 & Estacionária \\
\hline 29 Vales e Montanhas & $-2,35(-10,6 ; 6,6)$ & 0,494 & Estacionária \\
\hline 30 Vale da Luz & $-1,95(-16,1 ; 14,5)$ & 0,742 & Estacionária \\
\hline
\end{tabular}

Modelo de regressão de Prais-Winsten. Variável resposta: coeficiente anual de mortalidade por suicídio (por 100 mil habitantes). Variável explicativa: série temporal em anos, 2010-2016; ${ }^{*} p<0,05$ 


\section{DISCUSSÃO}

Os resultados deste estudo apresentaram algumas características epidemiológicas da mortalidade por suicídio no estado do RS de 2010 a 2016. Trata-se de um fenômeno eminentemente masculino, compreendendo quase $80 \%$ dos óbitos no período analisado e com coeficientes, em média, quatro vezes maiores do que os do sexo feminino. Esse padrão é também observado no Brasil ${ }^{6}$ e no mundo ${ }^{3}$, com exceção de alguns países orientais, como Bangladesh, China, Indonésia, Iraque e Paquistão, onde se encontram taxas mais altas entre as mulheres ${ }^{3}$.

Botega ${ }^{4}$ aponta que o desempenho do papel masculino envolve comportamentos mais agressivos e competitivos. Além disso, o abuso de bebidas alcoólicas pode deixar os homens mais impulsivos. As mulheres, em contrapartida, buscam mais ajuda e procuram tratamento em situações de sofrimento. Segundo Meneghel ${ }^{24}$, que problematiza as normas de gênero pautadas no patriarcado, os homens têm dificuldade em aceitar derrotas, perda de poder e de autoridade, bem como vivenciar situações tradicionalmente atribuídas às mulheres (dependência do cônjuge e adultério, por exemplo). Na velhice, a aposentadoria e as limitações para o trabalho podem provocar sentimentos de inutilidade, além do aparecimento de doenças e do declínio das funções vitais, especialmente a sexual. No mesmo sentido, Minayo' sugere que estudos compreensivos enfatizam as análises baseadas em gênero, "levando em conta as profundas inflexões culturais patriarcais e machistas que criam o ambiente microssocial opressor, responsável por desencadear eventos suicidógenos" (p. 226). Por outro lado, já é fato descrito na literatura que as mulheres empreendem mais tentativas de suicídio do que os homens ${ }^{2,6}$, originando diversas hipóteses que precisam ser mais bem estudadas para explicar o"paradoxo de gênero" no comportamento suicida 4 .

Em relação à faixa etária, os coeficientes de mortalidade mais altos foram observados entre pessoas idosas, padrão que se encontra também no restante do país ${ }^{6}$ e em quase todas as regiões do mundo ${ }^{3}$. Em contrapartida, $40 \%$ de todas as mortes se concentraram na faixa de 40 a 59 anos de idade. É preciso ressaltar o fato de serem homens e mulheres em idade produtiva, com significativos anos potenciais de vida perdidos ${ }^{25}$. Nesse sentido, Reichenhein e Werneck ${ }^{25}$ destacam que "a morte, quando ocorre numa etapa da vida de alta criatividade e produtividade, não só pune o próprio indivíduo e o grupo que lhe é próximo, mas também priva a coletividade de seu potencial econômico e intelectual" (p. 189). Nessa mesma coletividade, é encontrado um contexto de trabalho pautado na produção e em modelos de gestão que, muitas vezes, representam fatores de risco para o suicídio, conforme alerta a Organização Internacional do Trabalho ${ }^{26}$. Embora essa relação venha sendo estudada apenas contemporaneamente ainda de forma incipiente ${ }^{27,28}$, alguns autores apontam que o sofrimento psíquico derivado do trabalho pode se constituir em uma das causas do suicídio ou mesmo na causa principal ${ }^{28,29}$. Ainda nessa faixa etária, também conhecida como meia-idade, ganham destaque a perspectiva de envelhecimento e o período em que se sobrepõe o cuidado aos filhos e aos pais idosos ${ }^{30}$.

De modo geral, os coeficientes de mortalidade mantiveram-se estáveis no estado, com uma média de três suicídios a cada dia. Contudo, é necessário observar a tendência crescente entre mulheres de 10 a 14 e de 20 a 29 anos de idade. Meneghel et al. ${ }^{31}$ consideram a submissão a normas sociais, a presença de violência física ou sexual, os conflitos e os maus-tratos em relações conjugais como vulnerabilidades de gênero que "podem potencializar o suicídio" em mulheres (p. 212). Sabe-se que adolescentes encontram mais dificuldades para lidar com estresses agudos, como término de relacionamento e situações que provocam vergonha ou humilhação, por não possuírem plena maturidade emocional, sendo também mais propensos ao imediatismo e à impulsividade 4 . Nos Estados Unidos, a taxa de suicídio de meninas de $10 \mathrm{a}$ 14 anos de idade aumentou 3,4 vezes no período entre 1999 e $2017^{32}$. É possível tratar de uma interação entre as variáveis sexo feminino e faixa etária enquanto fatores de vulnerabilidade para o suicídio.

No que se refere à raça/cor, em consequência do processo histórico de imigração europeia no século XIX ${ }^{33}$, o RS tem mais de $80 \%$ da população autodeclarada branca ${ }^{18}$. A partir dessa base populacional, não surpreende a frequência majoritária de suicídios nesse grupo. Não obstante, também o coeficiente de mortalidade foi maior entre os caucasianos, fenômeno observado 
no Brasil ${ }^{6}$ e em outros países ${ }^{34}$. Apesar de o suicídio entre indígenas ser considerado um grave problema no Brasili, essa não é uma realidade do estado gaúcho.

No período analisado, os óbitos predominaram entre pessoas solteiras, viúvas ou separadas. O mesmo foi observado no país entre 2011 e 2015, quando as mortes de pessoas nessa situação conjugal alcançaram o dobro daquelas casadas ou em união estável ${ }^{6}$. Está documentado na literatura que o senso de isolamento e a falta de suporte social são fatores de risco para o suicídio ${ }^{3}$; entretanto, não se pode perder de vista que relacionamentos conflituosos também o são, assim como a violência de genêro ${ }^{3}$. Nesse sentido, chama atenção a pesquisa realizada no $\mathrm{RS}^{35}$ que encontrou maior proporção de óbitos entre os casados $(46,1 \%)$ nos anos de 1990 a 1998, evidenciando mudança nesse perfil ao longo de duas décadas. É possível notar que a situação conjugal registrada na declaração de óbito deve ser informada pelo familiar ou responsável ${ }^{36}$, tornando o dado mais fidedigno do que o estado civil.

Em relação à escolaridade, é preciso ressaltar o alto índice de "Não informada"e "Ignorada", que somaram $42,4 \%$ do total, comprometendo a análise dos dados. Por esse motivo, não foram calculados coeficientes. De todo modo, a maior parte das pessoas tinha até sete anos de estudo. Dados do Brasil, de 2011 a 2015, revelaram taxas mais altas de suicídio entre a população com essa mesma faixa de escolaridade 6 .

Enforcamento foi o método de perpetração mais utilizado (71\%), tanto por homens quanto por mulheres, o que também se observa no restante do país e no mundo ${ }^{3,6}$. Em pesquisa anterior, realizada no RS, foi encontrada uma proporção de $62,5 \%$ nos anos de 1980 a $1999^{35}$, o que indica um aumento de $8,5 \%$ na escolha por esse método ao longo da última década. $O$ fato de as mulheres utilizarem mais drogas e medicamentos do que os homens como método de perpetração possibilita tentativa de socorro, podendo explicar a maior proporção de óbitos ocorridos em hospital, quando comparadas aos homens. Medidas de restrição de acesso aos meios são indicadas pela OMS como importante estratégia para a redução das mortes por suicídio, especialmente no que diz respeito a armas de fogo, medicamentos e pesticidas, sendo mais difíceis de implementar quando se trata de enforcamento ${ }^{3}$.

Os meses com maior frequência de óbitos se concentraram no verão. Estudos internacionais ${ }^{37,38}$ já demonstraram aumento na incidência de suicídios associado à exposição solar, fato que ainda não é bem compreendido. Pesquisa realizada com dados países do hemisfério norte e do hemisfério sul (Austrália) encontrou um padrão sazonal nos casos de morte autoinfligida, com pico de incidência no início do verão (junho e dezembro, respectivamente), supondo que a luz solar poderia provocar um efeito desencadeador ${ }^{37}$. Da mesma forma, estudo realizado na Áustria, com dados de quase 70 mil casos, encontrou correlação entre duração de luz solar diária e frequência de suicídio, especialmente no dia da morte e até 10 dias antes ${ }^{38}$. Tais evidências levantam a hipótese de que o efeito da exposição ao sol no comportamento suicida seja mediado por substâncias cuja regulação é influenciada pela luz solar, como a melatonina e a serotonina, conhecidas por desempenharem importante papel na modulação do humor.

No tocante às regiões de saúde, os resultados encontrados indicam a necessidade de estudos aprofundados nas regiões Caminho das Águas, Botucaraí, Vale do Rio Pardo e Vales e Montanhas, que apresentaram os maiores coeficientes de mortalidade. Trata-se de regiões compostas majoritariamente por municípios com menos de 20 mil habitantes, muitos deles devotos à agricultura. Em todo o país, é constatado que a distribuição dos suicídios difere segundo o porte dos municípios, concentrando-se especialmente nos de menor número de habitantes ${ }^{2}$. Pesquisa realizada com dados de 2004 a 2010 observou que, nas regiões Sul, Sudeste e Centro-Oeste, as taxas de mortalidade por suicídio, ajustadas por idade, aumentaram à medida que o tamanho das cidades diminuiu ${ }^{39}$. Os autores presumem que, quanto menor 0 território, maior a possibilidade de se conhecer alguém que morreu por suicídio, sendo maior a possibilidade de que uma pessoa em situação de risco busque a mesma saída. Outro fator importante seria a menor cobertura de serviços de saúde em áreas mais afastadas ${ }^{39}$. No mesmo sentido, Minayo ${ }^{2}$ sugere como parte da explicação para esse padrão a maior capacidade de reconhecimento dos suicídios em cidades pequenas, onde as pessoas sabem o que acontece na comunidade, em contraposição ao anonimato das grandes cidades. Além disso, supõe que o 
fato possa estar relacionado à maior capacidade resolutiva dos serviços de saúde dos grandes centros, o que ajudaria a diminuir a letalidade das tentativas de suicídio.

Em relação aos agricultores gaúchos, Botega ${ }^{4}$ e $\mathrm{Cruz}^{9}$ apontam uma combinação de condicionantes que parece se associar às altas taxas de suicídio nessas localidades: a baixa escolaridade, a cultura patriarcal, o alcoolismo, a maior incidência de transtornos mentais, o histórico de suicídio nas famílias, a monocultura do tabaco das pequenas propriedades (dependentes de monopólios industriais), o endividamento e o potencial risco à saúde pelo uso de agrotóxicos. O Vale do Rio Pardo, onde se encontram municípios como Santa Cruz do Sul, Venâncio Aires e Candelária, é reconhecido pela forte presença da indústria do tabaco. Diversos estudos mostram que a exposição a agrotóxicos, em decorrência das alterações neurocomportamentais que podem provocar, constitui fator de risco para o suicídio, assim como a doença da folha verde, intoxicação aguda causada pela absorção da nicotina a partir do manuseio das folhas de fumo ${ }^{9}$. Ademais, estudos realizados nessa região 4,9 , de colonização predominantemente alemã, sugerem que, nesse sistema de referência cultural, a vida dedicada ao trabalho, a ausência de lazer, a perda de sentido com a interrupção da vida produtiva (sobretudo no caso de idosos) e o medo de ser um estorvo para os familiares, alinhados a um modo de ser com alto nível de exigência em relação a si próprio, podem constituir fatores de vulnerabilidade para o suicídio, especialmente diante de situações consideradas socialmente como fracasso ou desonra?.

Ainda em relação às regiões de saúde, também merecem especial atenção o Pampa e o Vale dos Sinos. Embora com taxas mais baixas que a média do estado no período, apresentaram tendência crescente de mortalidade.

Cabe ressaltar que este estudo utilizou apenas os dados referentes aos óbitos por suicídio confirmados, não incluindo aqueles codificados como eventos cuja intenção é indeterminada (categorias de $\mathrm{Y} 10$ a Y34, da CID-10) e sequelas de lesões autoprovocadas intencionalmente (Y87.0). Além disso, estima-se que, para cada morte entre adultos, haja de 10 a 20 pessoas que tentem tirar a própria vida ${ }^{3,40}$. Estudo multicêntrico de base comunitária coordenado pela OMS revelou que, em Campinas, mais de $17 \%$ das pessoas já haviam pensado seriamente em suicídio, quase $5 \%$ chegaram a elaborar um plano e $2,8 \%$ efetivamente tentaram o suicídio ${ }^{41}$. Sabe-se que uma tentativa prévia é o principal fator de risco isolado para a morte autoinfligida ${ }^{3}$, por isso também é necessário haver pesquisas que analisem os dados epidemiológicos desse comportamento. A notificação de violência autoprovocada passou a ser compulsória em $2011^{42}$, e os dados podem ser acessados por meio do Sistema de Informação de Agravos de Notificação (SINAN) ${ }^{43}$.

É possível apontar como limitação do presente estudo a utilização da população referente ao ano de 2015 no cálculo dos coeficientes de 2016, o que deve diminuir em algum nível as taxas do último ano. Ainda, os dados utilizados são secundários às declarações de óbito, documentos que apresentam, com frequência, falhas de preenchimento. Por fim, é reconhecido o sub-registro de óbitos por suicídio no estado no RS, assim como no Brasil e no mundo.

A análise de dados epidemiológicos subsidia as diversas esferas de gestão para o planejamento, a implantação, a avaliação e o aprimoramento das políticas de saúde ${ }^{44}$. Este estudo apresentou o perfil epidemiológico da mortalidade por suicídio no RS de 2010 a 2016, tornando evidente a magnitude do problema. Foram registrados 7.796 óbitos no período analisado, correspondendo a um coeficiente médio de 10,7 por 100 mil habitantes. Aproximadamente $80 \%$ dos suicídios foram de homens e $40 \%$ do total se concentrou entre 40 e 59 anos de idade. Os coeficientes mais altos foram encontrados entre pessoas idosas. Foi verificada tendência de mortalidade crescente para mulheres e decrescente para homens na faixa etária de 20 a 29 anos. Entre 10 e 14 anos de idade, foi identificada tendência crescente para o sexo feminino.

Tendo em vista que o suicídio é também expressão do modo de vida daqueles que morrem ${ }^{45}$, só é possível compreendê-lo em sua complexidade considerando a cultura e o contexto social. O presente estudo aponta para a necessidade de outras pesquisas que atentem para as combinações de variáveis próprias de cada região, principalmente aquelas em que as taxas de mortalidade são altas (centro e norte do estado) ou apresentam tendência crescente. 
É preciso ressaltar que a série temporal analisada foi constituída do número mínimo de pontos (sete anos) ${ }^{23}$, havendo a necessidade de análises de séries maiores e de forma permanente, com o propósito de identificar possíveis mudanças no padrão de comportamento do fenômeno.

Por compreender múltiplas dimensões - das quais se discutiu apenas uma parte -, o suicídio não pode ser abordado exclusivamente "como um problema individual, de ordem privada ou de forma reducionista, apenas como doença mental" (p. 228)'. Isto posto, é fundamental que as ações de prevenção se constituam em uma prioridade intersetorial, com ações articuladas entre a saúde e outras áreas, como educação, trabalho, assistência social e sistema judiciário, constando na agenda do poder público e da sociedade civil ${ }^{3,46}$.

\section{REFERÊNCIAS}

1. Minayo MCS. Suicídio: violência autoinfligida. In: Brasil. Ministério da Saúde, organizador. Impacto da violência na saúde dos brasileiros [Internet]. Brasília: Ministério da Saúde; 2005 [citado em 2018 ago 18]. Disponível em: http://bvsms.saude.gov.br/bvs/publicacoes/impacto_violencia.pdf

2. Minayo MCS, Assis SG, Souza ER, Correia BSC, Pacheco ML, Delgado PGG. Suicídios no Brasil: Mortalidade, tentativas, ideação, comportamento autopunitivo e prevenção. Brasília: Organização Pan-Americana da Saúde; 2010.

3. World Health Organization. Preventing suicide: a global imperative [Internet]. Luxembourg:WHO;2014 [citado em 2018 ago 18]. Disponível em: http://apps.who.int/iris/bitstream/10665/131056/8/9789241564878_eng. pdf?ua $=1 \& u a=1$

4. Botega NJ. Crise suicida: avaliação e manejo. Porto Alegre: Artmed; 2015.

5. Brasil. Ministério da Saúde. Ministério da Saúde lança Agenda Estratégica de Prevenção do Suicídio. Ministério da Saúde [Internet], Brasília, 2017 [citado em 2018 ago 18]. Disponível em: http:// portalarquivos2.saude.gov.br/images/pdf/2017/setembro/21/Coletiva-suicidio-21-09.pdf

6. Brasil. Ministério da Saúde, Secretaria de Vigilância em Saúde. Perfil epidemiológico das tentativas e óbitos por suicídio no Brasil e a rede de atenção à saúde. Ministério da Saúde [Internet], Brasília, 2017 [citado em 2018 ago 18]. Disponível em: http://portalarquivos2.saude.gov.br/images/pdf/2017/setembro/21/2017025-Perfil-epidemiologico-das-tentativas-e-obitos-por-suicidio-no-Brasil-e-a-rede-de-aten--ao-a-sa--de. pdf

7. Brasil. Ministério da Saúde, Fundação Nacional de Saúde. Manual de procedimento do sistema de informações sobre mortalidade. Ministério da Saúde [Internet], Brasília, 2001 [citado em 2018 ago 18]. Disponível em: http://svs.aids.gov.br/cgiae/admin/arquivos/sis_mortalidade.pdf

8. World Health Organization. Preventing suicide: How to start a survivors' group [Internet]. Geneva: WHO; 2008. [citado em 2018 ago 18]. Disponível em: http://www.who.int/mental_health/prevention/suicide/ resource_survivors.pdf

9. Cruz CW. As múltiplas mortes de si: suicídio de idosos no sul do Brasil [tese]. São Leopoldo: Repositório Digital da Biblioteca da Unisinos; 2014 [citado em 2018 ago 18]. Disponível em: http://www.repositorio. jesuita.org.br/handle/UNISINOS/3936

10. World Health Organization. Mental health action plan 2013-2020 [Internet]. Geneva: WHO; 2013 [citado em 2018 ago 18]. Disponível em: http://apps.who.int/iris/bitstream/10665/89966/1/9789241506021_eng. pdf?ua=1

11. Botega NJ, Werlang BSG, Cais CFS, Macedo MMK. Prevenção do comportamento suicida. PSICO. 2006;37(3):213-20.

12. Brasil. Portaria $n^{\circ} 1.876$, de 14 de agosto de 2006. Institui Diretrizes Nacionais para Prevenção do Suicídio, a ser implantadas em todas as unidades federadas, respeitadas as competências das três esferas de gestão. Diário Oficial da União [Internet], Brasília, 2006 [citado em 2018 ago 18]. Disponível em: http://bvsms. saude.gov.br/bvs/saudelegis/gm/2006/prt1876_14_08_2006.html

13. Brasil. Ministério da Saúde, Secretaria de Atenção à Saúde, Departamento de Ações Programáticas Estratégicas. Agenda de Ações Estratégicas para a Vigilância e Prevenção do Suicídio e Promoção da Saúde no Brasil: 2017 a 2020. Ministério da Saúde [Internet], Brasília, 2017 [citado em 2018 ago 18]. Disponível em: http://bvsms.saude.gov.br/bvs/publicacoes/acoes_estrategicas_vigilancia_prevencao_suicidio.pdf

14. Rio Grande do Sul. Decreto n 53.361, de 22 de dezembro de 2016. Institui Comitê de Promoção da Vida e de Prevenção do Suicídio. Diário Oficial do Estado [Internet], Porto Alegre, 2016 [citado em 2018 ago 18]. Disponível em: http://www.corag.rs.gov.br/doedia 
15. World Health Organization. Classificação Estatística Internacional de Doenças e Problemas Relacionados à Saúde - CID-10 [Internet]. São Paulo: Centro Colaborador da OMS para a Classificação de Doenças em Português (Centro Brasileiro de Classificação de Doenças). [citado em 2018 ago 18]. Disponível em: http:// www2.datasus.gov.br/cid10/V2008/cid10.htm.

16. Rio Grande do Sul. Secretaria de Estado da Saúde. Sistema de Informação de Mortalidade. Datasus [Internet], Brasília, 2018 [citado em 2018 ago 18]. Disponível em: http://200.198.173.165/scripts/tabcgi. exe?SIM/Obito

17. Brasil. Rede Interagencial de Informações para a Saúde. Estudo de estimativas populacionais por município, idade e sexo 2000-2015 - Brasil. Datasus [Internet], Brasília, 2018 [citado em 2018 ago 18]. Disponível em: http://tabnet.datasus.gov.br/cgi/deftohtm.exe?novapop/cnv/popbr.def

18. Instituto Brasileiro de Geografia e Estatística. Censo Demográfico [Internet]. 2018 [citado em 2018 ago 18]. Disponível em: https://sidra.ibge.gov.br/Tabela/136

19. Brasil. Decreto $n^{\circ} 7.508$, de 28 de junho de 2011. Regulamenta a Lei no 8.080, de 19 de setembro de 1990 para dispor sobre a organização do Sistema Único de Saúde - SUS, o planejamento da saúde, a assistência à saúde e a articulação interfederativa, e dá outras providências. Diário Oficial da União [Internet], Brasília, 2011 [citado em 2018 ago 18]. Disponível em: http://www.planalto.gov.br/ccivil_03/_ato2011-2014/2011/ decreto/D7508.htm

20. Instituto Brasileiro de Geografia e Estatística [Internet]. 2017 [citado em 2018 ago 18]. Disponível em: https://cidades.ibge.gov.br/brasil/rs

21. Rio Grande do Sul. Secretaria de Estado da Saúde. Resolução Comissão Intergestores Bipartite n 555 , de 19 de setembro de 2012. Secretaria da Saúde [Internet], Porto Alegre, 2012 [citado em 2018 ago 18]. Disponível em: http://www.saude.rs.gov.br/upload/arquivos/carga20170214/23101458-1348591506cibr555-12.pdf

22. Brasil. Rede Interagencial de Informações para a Saúde. Indicadores básicos para a saúde no Brasil: Conceitos e aplicações. Organização Pan-Americana da Saúde [Internet, Brasília, 2008 [citado em 2018 ago 18]. Disponível em: http://tabnet.datasus.gov.br/tabdata/livroidb/2ed/indicadores.pdf

23. Antunes JLF, Cardoso MRA. Uso da análise de séries temporais em estudos epidemiológicos. Epidemiol Serv Saude. 2015;24(3):565-76. http://dx.doi.org/10.5123/S1679-49742015000300024.

24. Meneghel SN, Gutierrez DMD, Silva RM, Grubits S, Hesler LZ, Ceccon RF. Suicídio de idosos sob a perspectiva de gênero. Cien Saude Colet. 2012;17(8):1983-92. http://dx.doi.org/10.1590/S1413-81232012000800009.

25. Reichenheim ME, Werneck GL. Anos potenciais de vida perdidos no Rio de Janeiro, 1990: as mortes violentas em questão. Cad Saude Publica. 1994;10(Suppl 1):188-98. http://dx.doi.org/10.1590/S0102$311 \times 1994000500014$.

26. International Labour Organization. Workplace stress: a collective challenge [Internet]. Turin: ILO; 2016 [citado em 2018 ago 18]. Disponível em: http://www.ilo.org/wcmsp5/groups/public/---ed_protect/--protrav/---safework/documents/publication/wcms_466547.pdf

27. Mendes JMR, Werlang R. Suicídio no meio rural no Rio Grande do Sul. In: Crespo AR, Bottega CG, Perez $\mathrm{KV}$, editores. Atenção à saúde mental do trabalhador: sofrimento e transtornos psíquicos relacionados ao trabalho. Porto Alegre: Evangraf; 2014. p. 133-60.

28. Merlo ARC, Heloani R. Suicídio (e trabalho). In: Vieira FO, Mendes AM, Merlo ARC, editores. Dicionário critico de gestão e psicodinâmica do trabalho. Curitiba: Juruá; 2013. p. 445-9.

29. Ceccon RF, Meneghel SN, Tavares JP, Lautert L. Suicídio e trabalho em metrópoles brasileiras: um estudo ecológico. Cien Saude Colet. 2014;19(7):2225-34. http://dx.doi.org/10.1590/1413-81232014197.09722013.

30. Côrte B, Lopes RGC, Silva ACL, Teixeira JB, Aguiar JS. Suicídio na envelhescência. Rev Latinoam Psicopatol Fundam. 2009;12(4):636-49. http://dx.doi.org/10.1590/S1415-47142009000400002.

31. Meneghel SN, Hesler LZ, Ceccon RF, Trindade AG, Pereira S. Suicídio de mulheres: uma situação limite? Athenea Digit. 2013;13(2):207-17. http://dx.doi.org/10.5565/rev/athenead/v13n2.1116.

32. Hedegaard H, Curtin SC, Warner M. Suicide mortality in the United States, 1999-2017. NCHS Data Brief [Internet]. 2018;(330):1-8 [citado em 2018 ago 18]. Disponível em: https://www.cdc.gov/nchs/data/ databriefs/db330-h.pdf

33. Herédia VBM. A imigração européia no século passado: o programa de colonização no Rio Grande do Sul. Scr Nova. 2001;5(94).

34. Krug EG, Dahlberg LL, Mercy JA, Zwi AB, Lozano R. editores. World report on violence and health [Internet]. Geneva:WHO; 2002 [citado em 2018 ago 18]. Disponível em: https://www.opas.org.br/wp-content/ uploads/2015/09/relatorio-mundial-violencia-saude.pdf 
35. Meneghel SN, Victora CC, Faria NMX, Carvalho LA, Falk JW. Características epidemiológicas do suicídio no Rio Grande do Sul. Rev Saude Publica. 2004;38(6):804-10. http://dx.doi.org/10.1590/S003489102004000600008 . PMid:15608898.

36. Brasil. Ministério da Saúde, Secretaria de Vigilância em Saúde, Departamento de Análise de Situação de Saúde. Manual de instruções para o preenchimento da declaração de óbito. Ministério da Saúde [Internet], Brasília, 2011. [citado em 2018 ago 18]. Disponível em:http://svs.aids.gov.br/download/manuais/ Manual_Instr_Preench_DO_2011_jan.pdf

37. Petridou E, Papadopoulos FC, Frangakis CE, Skalkidou A, Trichopoulos D. A role of sunshine in the triggering of suicide. Epidemiology. 2002;13(1):106-9. http://dx.doi.org/10.1097/00001648-200201000-00017. PMid:11805594.

38. Vyssoki B, Kapusta ND, Praschak-Rieder N, Dorffner G, Willeit M. Direct effect of sunshine on suicide. JAMA Psychiatry. 2014;71(11):1231-7. http://dx.doi.org/10.1001/jamapsychiatry.2014.1198. PMid:25208208.

39. Marín-León L, Oliveira HB, Botega NJ. Suicide in Brazil, 2004-2010: the importance of small counties. Rev Panam Salud Publica. 2012;32(5):351-9. http://dx.doi.org/10.1590/S1020-49892012001100005. PMid:23338692.

40. Brasil. Ministério da Saúde, Organização Pan-Americana da Saúde. Prevenção do Suicídio: Manual dirigido a profissionais das equipes de saúde mental. Ministério da Saúde [Internet], Brasília, 2006. [citado em 2018 ago 18]. Disponível em: http://bvsms.saude.gov.br/bvs/publicacoes/manual_editoracao.pdf

41. Botega NJ, Barros MBA, Oliveira HB, Dalgalarrondo P, Marín-León L. Suicidal behavior in the community: Prevalence and factors associated with suicidal ideation. Rev Bras Psiquiatr. 2005;27(1):45-53. http:// dx.doi.org/10.1590/S1516-44462005000100011. PMid:15867983.

42. Brasil. Ministério da Saúde. Portaria n 1.271, de 6 de junho de 2014. Define a Lista Nacional de Notificação Compulsória de doenças, agravos e eventos de saúde pública nos serviços de saúde públicos e privados em todo o território nacional, nos termos do anexo, e dá outras providências. Diário Oficial da União [Internet], Brasília, 2014 [citado em 2018 ago 18]. Disponível em: http://bvsms.saude.gov.br/bvs/ saudelegis/gm/2014/prt1271_06_06_2014.html

43. Rio Grande do Sul. Secretaria de Estado da Saúde. Sistema de Informação de Agravos de Notificação. Datasus [Internet], Brasília, 2018 [citado em 2018 ago 18]. Disponível em: http://200.198.173.165/scripts/ deftohtm.exe?snet/violencianet

44. Brasil. Ministério da Saúde, Secretaria de Vigilância em Saúde. Instrutivo: notificação de violência interpessoal e autoprovocada. Ministério da Saúde [Internet], Brasília, 2015 [citado em 2018 ago 18]. Disponível em: http://portalarquivos.saude.gov.br/images/pdf/2016/fevereiro/16/instrutivo-ficha-sinan5-1--vers--o-final-15-01-2016.pdf

45. Cruz CW, Fernandes NF, Wolkmer AN. Observatório de análise de situação do suicídio no RS: Um breve histórico. In: III Seminário Internacional de Políticas Públicas, Intersetorialidade e Família [Internet]; 2017; Porto Alegre, BR. Porto Alegre: PUCRS; 2017 [citado em 2018 ago 18]. Disponível em: http://ebooks.pucrs. br/edipucrs/anais/sipinf

46. World Health Organization. Public health action for the prevention of suicide: a framework [Internet]. Geneva: WHO; 2012 [citado em 2018 ago 18]. Disponível em: http://apps.who.int/iris/ bitstream/10665/75166/1/9789241503570_eng.pdf 